\title{
CUADRILLAS EN EL PAIS VASCO: IDENTIDAD LOCAL Y REVITALIZACION ETNICA
}

\section{Eugenia Ramírez Goicoechea}

Nadie que haya estado con frecuencia en el País Vasco y conozca mínimamente su entramado social duda del papel tan importante como curioso que tienen las cuadrillas de amigos en la estructura de relaciones interpersonales.

Lo que aquí se pretende es esbozar las líneas generales de un argumento que defiende el papel de este tipo de grupo informal en el proceso de revitalización étnica del País Vasco.

En primer lugar, y como línea de análisis, sin ahondar excesivamente en el origen histórico de esta peculiar forma de sociabilidad y asociacionimo, trataremos de analizar qué representa la cuadrilla en la estructura de relaciones personales de la cultura euskaldún y cuáles son sus potencialidades en cuanto a movilización de recursos humanos, de información, de aglutinación y cohesión, etc. Por último, y directamente derivado de lo anterior, analizaremos el papel operado por las cuadrillas como dinamizadoras y catalizadoras de un proceso político y cultural de revitalización étnica.

Una investigación histórica de los orígenes de la cuadrilla pasaría por el análisis del sistema tradicional de defensa y policía urbana de las villas, que se hacía por cuadrillas o cuarteles ${ }^{1}$ fundamentadas en la identidad de barrio, así como por el estudio de la cofradía tradicional, tanto gremial como religiosa (como vivencia de la localidad). El origen de las sidrerías y posteriormente de las sociedades gastronómicas tcompletaría el panorama de aproximación histórica a la emergencia de este tipo de grupo informal.

1 Arpal, J., "Solidaridades elementales y organizaciones colectivas en el País Vasco (cuadrillas, txokos, asociaciones)", en Euskal Herriko Soziologiazko Ikastaroa, Cuaderno de Formación, núm. 3, Bilbao, IPES, p. 55. 
De lo que no cabe duda es de que aparece ligado al proceso de industrialización y urbanización del País Vasco y a la reformulación de las antiguas formas comunitarias de relación y reciprocidad ${ }^{2}$. Por tanto, a pesar de su arraigo y extensión, podemos decir que es un fenómeno totalmente moderno.

En términos socioantropológicos, ¿cómo podemós definir a la cuadrilla? En principio, suponiendo un «continuum» a nivel organizativo entre lo más formalizado y lo menos formalizado, diríamos que a la cuadrilla puede considerársela como un grupo informal (no tiene estatutos ni «reglas de juego» explícitas), entendiendo éste como conjunto de personas que se comunican todas ellas directamente «face-to-face» con las demás, que son interdependientes en cuanto a necesidades, objetivos y afinidades ${ }^{3}$ y que no tienen formalizados explícitamente sus principios normativos, siendo que, por ende, las pautas de conducta esperadas depeden mucho de las peculiaridades de sus miembros ${ }^{4}$. En este contexto, las situaciones y las posiciones no suelen estar claramente entructuradas. Y todo ello porque la cuadrilla, como grupo informal (cuya «continuación» es una escala de formalización sería la «peña», la «sociedad popular») representa la cristalización de las relaciones personales de amistad, con la espontaneidad e inestructuración que éstas conllevan.

Como tipo específico de grupo informal, debido a su fundamental característica de grupo de amigos, a su composición y a su nacimiento, suele asemejarse a los grupos de pares o iguales. Y esto en un doble sentido. La cuadrilla ${ }^{5}$ está formada por un grupo variable de varones, generalmente de edades parecidas y cuya génesis viene determinada por la vecindad común en la mayoría de los casos, reforzada por la escolaridad común o la prestación del servicio militar al mismo tiempo e incluso a veces en el mismo lugar.

¿Cuáles son los espacios/tiempo propios de la cuadrilla? Antes dijimos que éste es un grupo estrechamente ligado al surgimiento y sobre todo extensión de las formas de vida urbanas. Es, por tanto, la calle el medio principal en el que se desenvuelve, entendiendo ésta en un sentido amplio. Lugar privilegiado de la espontaneidad frente a la rigidez de lo organizativo, la calle es el espacio social de lo cotidiano por excelencia, de la circulación y de

2 Caro Baroja, J., Los vascos, IV ed., Istmo, Madrid, 1980, p. 225; Echegarya, B. de, "La vecindad. Relaciones que engendra en el País Vasco", Revista Internacional de Estudios Vascos, XXIII, 1932; Douglas, W., Muerte en Murélaga. El contexto de la muerte en el País Vasco, Barral Ed., Barcelona, 1973, cap. 4.

${ }^{3}$ Nos referimos en cuanto a los aspectos de comunicación, ocio, etc.

4 Cfr. Homans, G. C., The Human Group, Routledge and Kegan Paul, London, 1951, cap. I; ShERIF, M., "The concept of Reference Group in Human Relations", en Herbert Hyman and Eleanor Singer, eds., Readings in Reference Group Theory and Research, The Free Press, New York, 1968, p. 93; MaIER, N. R. F., "Ventajas e inconvenientes de la solución de problemas de grupo", y Friedrer, F. E., "El estudio del liderazgo: el modelo de la contingencia", en José R. Torregrosa, Teorías $e$ investigación en la psicología social actual, C.I.S., Madrid, 1974, pp. 843 y 912, respectivamente; Hare, A. P.; Borgati, A. E. F., y Bales, R. F., eds., small Groups, II, Ed. Knopf, New York, 1965.

s En su forma tradicional, prototípica y como grupo "aislable" antropológicamente. 
la comunicación ${ }^{6}$. Aquí, la calle, más que como espectáculo, es concebida como lugar de interacción, de «toma de la ciudad» y de aprehensión de sus circuitos y redes de intercambio simbólico. Y para ello el «txikiteo» es el vehículo/escusa de esta apropiación espacio-temporal de la calle. Espacial por la presencia física de las cuadrillas y por el recorrido que realizan de la calle (en sentido estricto) y de los bares, y temporal porque se «domina» y controla el tiempo abstracto por medio de la rutinización de las actividades, del tiempo concreto/significante que se destruye ${ }^{7}$.

Es aquí, en la acción/interacción con el medio (el espacio social y físico de la comunidad, los otros...), en el «poteo», cuando el grupo, a efectos de identificación, se distingue especialmente, cobra todo su sentido, y no tanto en el bar/lugar de reunión que tienen casi todas las cuadrillas como «cuartel general», sobre todo si viven en un barrio alejado del centro de la ciudad (los Cascos Viejos suelen ser los lugares privilegiados de poteo). En esta interacción con el medio hay que destacar que no existe, por lo común, una relación específica cuadrilla/cuadrilla. El medio, los otros, es contemplado como un agregado difuso, sin estructuración propia. La relación de la cuadrilla con «los otros» lo es de uno de sus miembros con un individuo concreto, conocido o amigo, que, normalmente y en ese momento, no es contemplado como miembro de otra cuadrilla ni como su «tepresentante», sino sólo como indiviualidad específica.

Son muchas las características que distinguen una cuadrilla de otra, aparte de su composición y el número de sus miembros: su lugar de reunión o «cuartel general», los bares que frecuenta y la ruta seguida, el tipo de diversión que prefiere (pelota, cine, montaña...), así como la afinidad ideológica y el grado de politización de sus actividades y conversaciones. Porque ocurre con frecuencia que sus miembros tengan parecidas ideas políticas o incluso que sus votos se dirijan al mismo partido. Entre cuadrillas de «viejos», la homogeneidad ideológica es menor y a menudo el último tema de conversación del que se habla es el de la política. Esto no ocurre en absoluto con la mayoría de cuadrillas que han vivido intensamente la década de los años 70. Pero de todo esto hablaremos más adelante. Lo que es importante destacar es que esta forma de sociabilidad aparece, en sus orígenes, ligada a las clases medias, permeando con el tiempo y hacia abajo a otras capas sociales. La componente de clase de algunas cuadrillas está en relación con la afinidad económica y sacial de los miembros de un mismo barrio.

El tipo de integración en la cuadrilla, como en todo grupo informal, es relativamente espontáneo. La integración puede decirse que es solidaria-mecánica, siendo tácitas las normas y condiciones de pertenencia y participación.

- Lefebvre, H., De lo rural a lo urbano, antología por Mario Gaviria, II Ed., Península, Barcelona, 1973, p. 94.

1 Lalive d'Epinay, Ch., "La vie quotidienne. Essai de construction d'un concept sociologique et anthropoligique", Cahiers Internationaux de Sociologie, LXXIV. Nouvelle Série, 30ième année, Janvier-Juin 1983, París. 
La condición de miembro se refuerza día a día a través de la práctica común (el «txikiteo», pero también a través de las otras actividades que lleva a cabo el grupo, que suelen ser, en su mayoría, recreativas, pues la cuadrilla no es un «grupo de trabajo»). El abandono puede ser difuso y procesual, como el que se deriva del no poder o no querer tomar parte en estas actividades y prácticas habituales. En el primer caso, un motivo bastante común es el de estar en paro laboral y no poder seguir «el tren de vida» de la cuadrilla, en lo que a gastos se refiere. En estos casos, el abandono suele ser temporal, reintegrándose el sujeto en cuanto dispone de medios económicos para pagar la «ronda» correspondiente o la cena "de los viernes», por ejemplo. En el segundo, los motivos pueden ser muy varios. Uno de ellos, y a menudo el fundamental, es el de «echarse novia» o casarse. En este caso, el sujeto puede reservar un día a la semana para estar con los amigos, pero sin seguir el ritmo semanal de éstos. La salida del grupo puede tener también un carácter brusco y repentino, debido quizá a la falta de cumplimiento grave de una obligación de solidaridad o una toma de postura puntual ante un hecho concreto que no se corresponde con la «ideología oficial» y los valores del grupo. Nos referimos a los casos de escisión de una cuadrilla como reflejo de divergencias surgidas en algún grupo político con el que estaban ligados ideológicamente. De todos modos, las «expulsiones» como tales son raras (no olvidemos que se trata de un grupo informal). Más bien al individuo en concreto «se le irá dando poco a poco de lado hasta que se dé cuenta y no vuelva».

El tipo de relación del miembro con respecto al grupo como totalidad suele ser bastante gregaria en algunos aspectos, cosa que es dada, en cierta manera, por las condiciones de origen de la cuadrilla y por sus características concretas de actuación: se sabe siempre dónde encontrar al grupo en una hora determinada, debido a la rutinización de la que hablábamos antes, hay poca promoción del factor individual como tal, siendo los fenómenos de liderazgo poco concretos ${ }^{8}$. Por otra parte, la relación entre los miembros de la cuadrilla no suele ser muy íntima en cuanto a comunicación de problemas personales. El individuo, normalmente, tiene sus mejores amigos (dos o tres) entre los miembros de la cuadrilla, y es con éstos particularmente con los que más profundamente se comunica a nivel de vivencias, problemas y preocupaciones personales, y no con la cuadrilla como totalidad. Esta comunicación se realiza generalmente por medio de los subgrupos que se forman en la «práctica» del «txikiteo», tanto en el recorrido de la calle como en la ocupación del bar. Por tanto, como vemos, dentro de la cuadrilla existen varias «cliques» que priman la relación del individuo con respecto a determinados miembros del grupo.

También queremos señalar el papel como grupo de referencia positivo que desempeña la cuadrilla para el individuo, tanto desde el punto de vista nor-

${ }^{3}$ Cfr. Schachter, S., Psicología de la afiliación. Estudios experimentales de las fuentes del gregarismo, Paidós, Buenos Aires, 1966, p. 13. 
mativo, de conducta, así como punto de referencia para comprar y contrastar (hacer juicios y evaluaciones) y como lugar social al que se aspira a entrar o seguir siendo aceptado)'.

Si antes hemos hablado de la acción como elemento diacrítico de la cuadrilla en su praxis cotidiana en la esfera pública, en la calle, podemos decir que en el «sistema interno» del grupo, considerando éste desde el punto de vista de las relaciones de sus miembros entre sí ${ }^{10}$, lo fundamental es la interacción, que suele ser bastante estrecha (aunque no especialmente íntima, como hemos visto) y que genera fuertes sentimientos de pertenencia, fundamentáles para la aparición de la necesaria cohesión de todo grupo. Esta interacción entre los miembros del grupo se lleva a cabo a través del «poteo» como sistema de comunicación y por medio también de todo un sistema de contraprestaciones personales mutuas de favores, que pueden ir desde prestar el coche o dinero, ayudar a alguna fanea, hasta la visita al hospital o a la cárcel.

Lo que es fundamental destacar es que la cuadrilla es un grupo mediador entre la familia, como grupo primario, y otro tipo de grupos y esferas de actividad e instituciones, también grupos de referencia del individuo. Por tanto, por una parte, cumple una función en cuanto a eje intermediario, en su carácter intersticial, entre estas dos esferas de actividades y relaciones, $y$, por otra, ejerce de mecanismo de integración en la comunidad local frente a lealtades más extrañas y lejanas. Es el retículo, en el sentido que le da Henri Lefebvre ${ }^{11}$, en cuanto que sitúa «a los grandes grupos en la cotidianidad y recíprocamente» a través de la información y comunicación social «incontrolada» y del ascendiente que tiene el grupo sobre sus miembros ${ }^{2}$. En este sentido hay que destacar el papel de la cuadrilla como medio de difusión de toda una información tanto local como externa. A través de la reelaboración de los contenidos informativos de los grandes medios de comunicación de masas, a través de la comunicación intersubjetiva, la información anónima de estos «mass-media» se vuelve personal, concreta y significante para los individuos, que, de este modo, realizan un consumo muy mediatizado y matizado de estos medios de comunicación de masas.

Es ésta, a nuestro entender, una de las fundamentales dimensiones estructurales de la cuadrilla en la vida comunitaria del País Vasco. De ahi su importancia y extensión como base, como modelo y stock de pautas de conducta de otros tipos de asociacionismos relativamente más estructurados (sociedades recreativas, gastronómicas, etc.).

"Shibutani, T., "Reference Groups as Perspectives", en Herbert H. Hyman and Eleanor Singer, eds., op. cit., pp. 103-114.

"Homans, G. C., op. cit., caps. V y VI.

"LefEBve, H., op. cit., pp. 100 y 101.

12 Según Xabier EDER, hablando sobre Navarra y que podemos aplicar al resto del País Vasco, “... la información veraz se sigue transmitiendo in fide tertulistarum'..." ("Un reino mecido a ambos lados del Pirineo", "El Pais", 29-4-83). 
Esta dimensión estructural se ve reforzada por la característica de agente socializador que suele tener el grupo de iguales. Dentro de lo que podríamos considerar la socialización informal (el medio familiar, el medio comunitario) frente a los medios y agentes de la socialización formal (escuela, maestros, libros, etc.), la cuadrilla, considerada como grupo de iguales, facilita y promueve los mecanismos y contenidos de identidad local ${ }^{13}$, donde las cuadrillas de «mayores» funcionan indirectamente como modelos, como grupos de referencia. Es en este medio donde se vivencian muchos de los valores y pautas de la comunidad, como puedan ser: la segregación de las mujeres en ciertas esferas de «lo masculino», lo grupal como modelo de relación, la camaradería como sistema de obligaciones mutuas, la calle como espacio social público por excelencia, pero no anónimo, donde las cuadrillas pueden ejercitar e imprimir su idiosincrasia particular a espacios comunitarios muy concretos, la fiesta como expresión colectiva fundamental, los deportes autóctonos como rasgos de identidad, etc., etc. $Y$ todo esto en una edad fundamental para el sujeto, la adolescencia - edad, por otra parte, de la formación de las cuadrillas-, donde aprende a irse desenvolviendo en los valores y pautas de interacción de su comunidad.

Por tanto, y dentro de un proceso social general de recomunitarización de las relaciones intersubjetivas en el País Vasco, la cuadrilla, a través de sí misma y de todas sus concreciones organizativas (peñas, sociedades, etc.), facilita la integración local del individuo por medio de la creación de una identidad propia, de modo que éste vive de una manera privilegiada su propio entorno comunitario.

Pero no sólo queremos destacar las cuadrillas como mecanismos de integración comunitaria, sino como mecanismos de recepción, recreación y reformulación de contenidos de diferenciación cultural en el marco de un proceso de revitalización étnica, sobre todo gracias a su posición estratégica intersticial en la vida cotidiana de la comunidad. Estas redes, este entramado de la sociedad civil, se han convertido, sobre todo en un momento histórico muy concreto, en lugares privilegiados de recepción y amplificación de un código simbólico étnico «nosotros-ellos», delimitando y definiendo claramente espacios y criterios de pertenencia diferenciada ${ }^{14}$. Estas cuadrillas se han convertido, sobre todo en la época de los años 70 , en auténticos dinamizadores étnicos. Por sí mismas y por medio de las asociaciones colectivas de las que pudieran formar parte, por medio de las actividades de éstas (manipulación de símbolos - bandera, himno, etc- cultivo y promoción del euskera, organización de fiestas, actividades culturales y deportivas, marchas, etc.), las cuadrillas han colaborado de una manera fundamental al refuerzo de la conciencia

13 Ramirez Goicoechea, E., "Procesos educativos y cultura en el País Vasco", Madrid, 1984.

is BARTH. F., Introducción. en Fredrick Barth, comp., Los grupos étnicos y sus fronteras, FCE, México, 1976, pp. 9-49. 
vasca ${ }^{15}$. Todos recordamos como, en la época franquista, el deporte, la fiesta, las gastronomía, los bares, eran espacios de expresión y reivindicación políticas bajo el escudo de la reivindicación cultural. Eran la expresión de la «nación prohibida» ${ }^{16}$. Aquí, la política toma el lenguaje de la cultura y viceversa, reforzándose mutuamente y constituyendo todo un movimiento que hemos dado en llamar de reivindicación étnica.

En este sentido queremos especificar qué entendemos por el concepto 'etnicidad'. La noción que estamos utilizando no es una noción de etnicidad que enfatiza sólo la creación de límites y de criterios de autoadscripción subjetivos ${ }^{17}$, sino la de un discurso social determinado que quiere recuperar/recrear determinados factores «objetivos» de diferenciación cultural, elegidos arbitrariamente y que considera como diacríticos en términos de inclusión/exclusión, con el objeto de aglutinar ciertos colectivos en un provecto social y de poder concreto. En este sentido, etnicidad es una construcción social en e] tiempo, un proceso que implica una relación estrecha en la reivindicación cultural y la reivindicación política y que no sólo tiene como referente último a «los otros», sino al Estado/Nación en el cual el grupo étnico (portador de la reivindicación de tal tipo) está inserto ${ }^{16}$.

Este concepto de etnicidad nos parece pertinente para el caso vasco, porque la identidad cultural es fundamentalmente vivida/manipulada/expresada como identidad nacional ${ }^{19}$, implicando en su trasfondo un proyecto político alternativo al poder «oficial».

Lo que se ha dado en llamar la falta de legitimidad del Estado español en Euskadi ${ }^{20}$ y la represión indiscriminada del franquismo respecto de cual. quier atisbo de manifestación cultural propia o disidencia política o ideológica, provocó una reacción de rechazo muy fuerte hacia todo tipo de poder institucional o no que viniera «de Madrid». Esta oposición se refugió en las redes de la sociedad civil, representadas por las cuadrillas tanto a nivel cotidiano

"Cfrr. Langaik, núm. 3, agosto 1983, "Debate sobre la conciencia vasca en Navarra", IPES, Navarra, pp. 21 y 24.

10 GurrutXaGa, A., "La producción de la conciencia nacional" en Euskal Herriko Sociologiazko Ikastaroa, op. cit., p. 83: Núñez-Astrain, L. C. La sociedad vásca actual, San Sebastián, Txertoa. 1977, cap. I.

17 Cfr. Bath, F., op. cit.

1* Cfr. Cohen, A., Two Dimensional Man. An Essay on the Anthropology of Power and Symbolism in Complex Society, University of Chicago Press, Berkeley and Los Angeles, 1974, p. 97.

19 Queremos recalcar que si puede considerarse a la identidad cultural como identidad nacional, sólo es en el caso de conformar a la primera bajo un proyecto político, un "deseo de Estado" en el decir de Juan Aranzadi "Cfr. Milenarismo vasco. Edad de Oro, etnia y nativismo, Taurus Ed., Madrid, 1982, p. 86).

30 Cfr. PÉrez-Agote, A., "La identidad colectiva: noción sociológica y dimensión política. Para una visión cualitativa de nuestra encuesta", en J. A. Garmendia, F. Parra Luna y A. Pérez-Agote, Abertzales y vascos. Identificación vasquista y nacionalista en el Pais Vasco, Akal, Madrid, 1982, pp. 27, 28 y 30; Gurrutxaga, A., op. cit., p. 79 y ss.; Bidart, P., "Politique, culture et science", en Pierre Bidart, dir., La nouvelle société Basque. Ruptures et changements, L'Harmattan, Paris, 1980, página 10. 
como a nivel festivo, extraordinario ${ }^{21}$. De este modo, y sobre todo en medios urbanos y semiurbanos, la cuadrilla de amigos se reforzó como espacio de socialización política en muchos casos, como vehículo de politización de la vida cotidiana, de movilizaciones y capacidad de convocatoria, dando a lo político, entre otras causas, ese carácter de inmediatez que ha renido en el País Vasco. En este sentido, la «permeabilidad" política de la cuadrilla, a efectos de la estructura interna del grupo, depende de la participación directa de alguno de sus miembros en un grupo político determinado, así como en el apoyo ideológico y afectivo de los miembros a una opción política determinada.

Estas han permitido que el partido, el grupo político, entonces prohibido y clandestino, llegara directamente a la vida cotidiana, a los individuos ${ }^{22}$ y les implicara personalmente, movilizándolos en la defensa de una identidad colectiva amenazada cultural y físicamente. En nuestra opinión, la lucha política en Euskadi no se hubiera convertido en todo un movimiento social si no hubiera adoptado un lenguaje «cultural», si no hubiera apelado a una identidad cultural y si no hubiera utilizado los mecanismos de integración local y comunitaria que son estos grupos intersticiales y la intensidad de relaciones intersubjetivas que recrean.

Para terminar, queremos señalar que esta estrecha interrelación vida cotidiana/identidad cultural/política es fruto de un determinado momento histórico en la vida social del País Vasco. Podemos afirmar que a partir de la legalización de los partidos y actividades políticas, las cuadrillas se han despolitizado de alguna manera, a pesar de que existan algunos casos todavía de fuerte ideologización política. La aparición de los canales propios de expresión política (periódicos y medios de comunicación en general, campañas electorales, etc.) está sustituyendo progresivamente a la relación personal intersubjetiva como vehículo de información/comunicación y actualización políticas. En cuanto a la relación identidad cultural/política, esta última se sigue haciendo en nombre de «los valores esenciales de la cultura euskaldún». No obstante, las manifestaciones culturales, las fiestas, han dejado de ser foro político privilegiado. En esta «traslación» de lo político, condicionada por la aparición de nuevos espacios sociales, no hay que olvidar la despolitización real que se observa actualmente en las nuevas generaciones, cuya atención se dirige hacia otros intereses. No obstante, por el momento, la cuadrilla sigue siendo lugar de reencuentro y de vivencia de la identidad local, aunque, también hay que decirlo, está sufriendo una serie de transformaciones que la van alejando de su forma tradicional en cuanto a composición y actividades.

2 Cfr. Garcia tabuenca, A.; Gaviria, M.: Tuñón, P., et all., El espacio de la fiesta y la subversión. Análisis socioeconómico del Casco Viejo de Pamplona, Hórdago, San Sebastián, 1979, cap. V.

${ }^{22}$ Cfr. White, W. F., Street Corner Society. The Social Structure of an Italian Slum, Iled., The University of Chicago Press, Chicago, 1965, p. 217, y CoHeN, A., op cit., p. 59. 
CRITICA DE LIBROS 